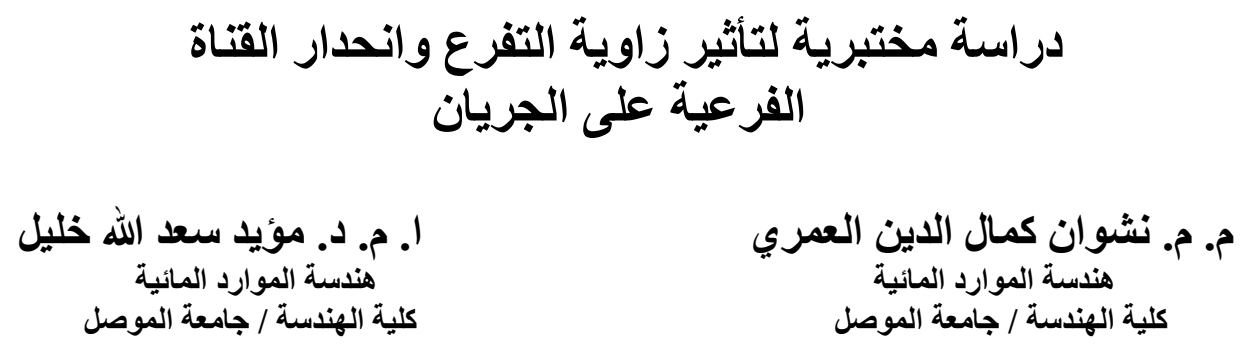

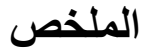

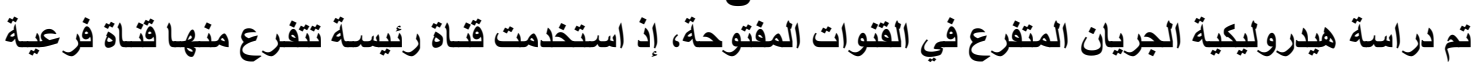

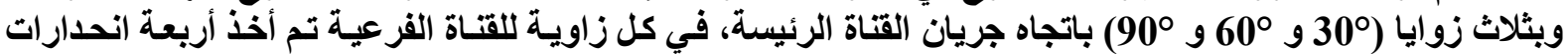

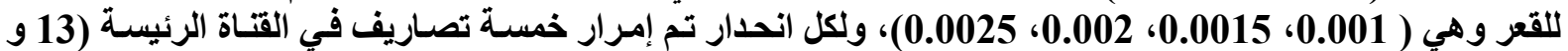

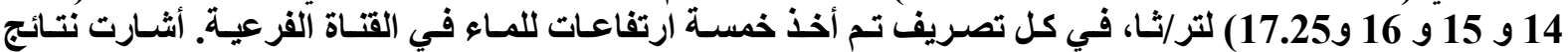

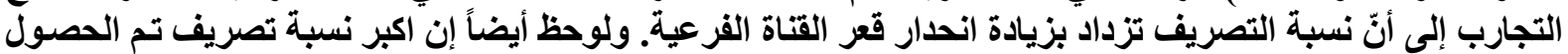

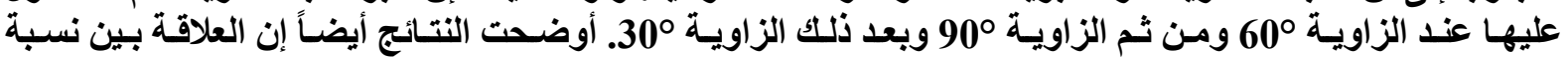

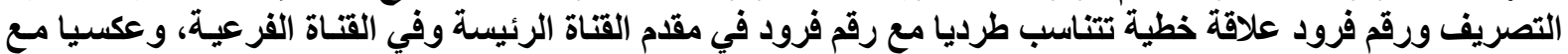

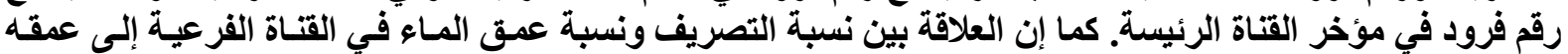

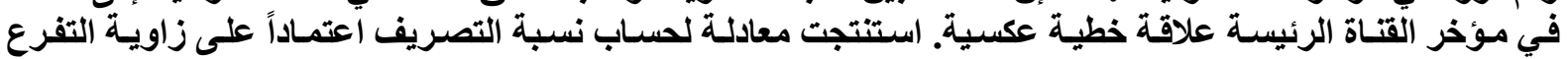

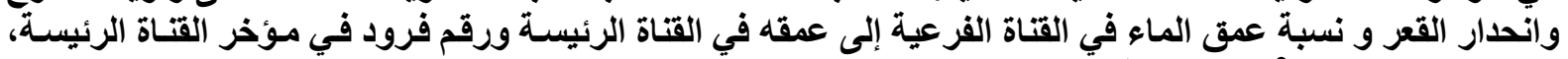
وبلغ معامل التحديا (
\end{abstract}

\title{
Laboratory Study of the Effect of the Branching Angle and the Branching Channel Slope on Flow
}

\author{
Nashwan K. Al Omari \\ Water Resources Engineering \\ Dr. Moayed S. Khaleel \\ College of Engineer / Mosul University
}

\begin{abstract}
In this paper the hydraulic characteristics of branching flow in open channel has been studied, a main channel was used with a branching channel connected with three angles $\left(30^{\circ}, 60^{\circ}, 90^{\circ}\right)$ with four bed slopes $(0.001,0.0015,0.002,0.0025)$ and at each slope five values of discharge were pass in the main channel $(13,14,15,16$ and 17.25) $1 / \mathrm{s}$. For each discharge five depths of water were allowed in the branching channel in each experiment. The results of the experiments indicated that the discharge ratio increases with increases bed slope of the branching channel. It was also found that the highest discharge ratio is obtained in the angle $60^{\circ}$ then the angle $90^{\circ}$ and then angle $30^{\circ}$. The results also showed that the relationship between the discharge ratio and Froude number is linear and direct proportional to the Froude number in the upstream main channel and in the branching channel, and inversely with the Froude number in the downstream main channel. The relationship between discharge ratio and the ratio of water depth in the branching channel to water depth in the downstream main channel is linear and inverse. An empirical equation to calculate the discharge ratio was observed, with a coefficient of determination $\left(R^{2}\right)$ equal $(0.953)$.

Key Word: Branching channel, Branching channel slope, open channel, Branching flow and Branching angle.
\end{abstract}


المقدمة

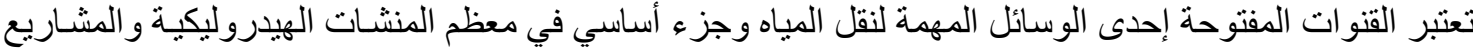

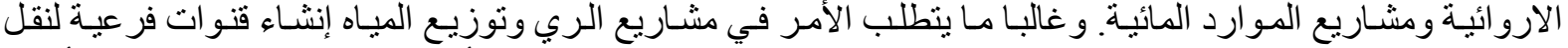

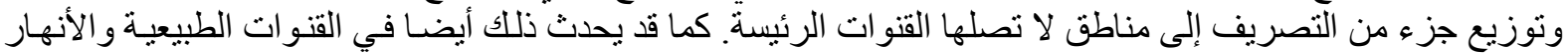

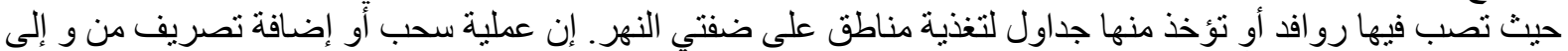

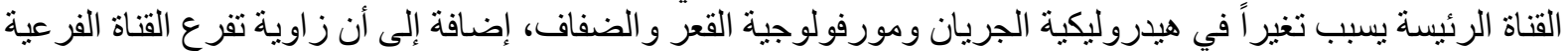

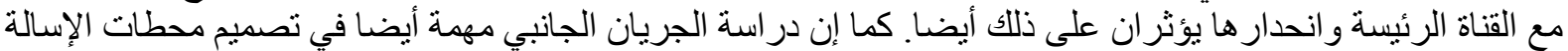

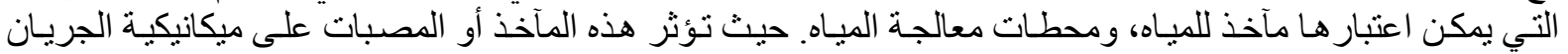

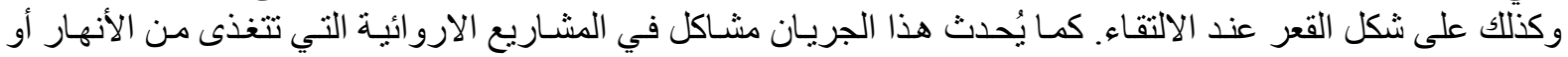

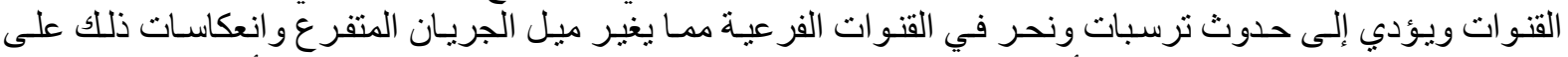

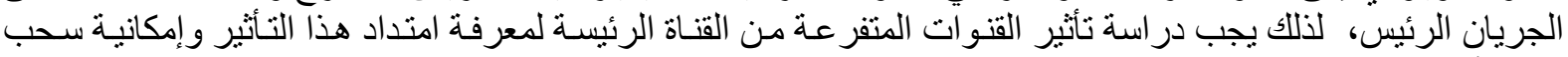

فروع أخرى من القناة الرئيسة.

الار اسنات السابقة

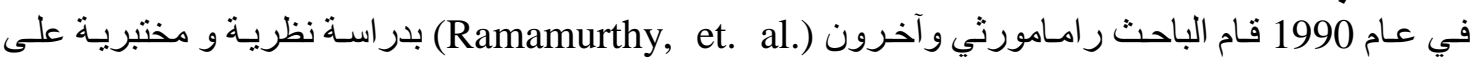

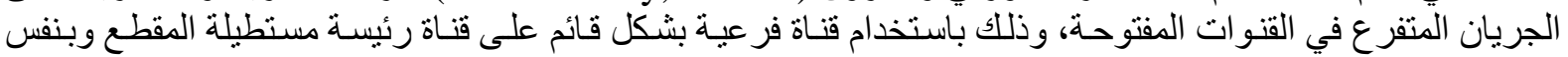

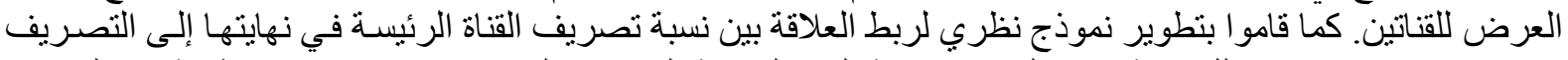

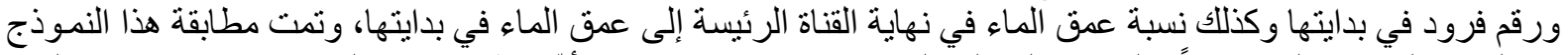

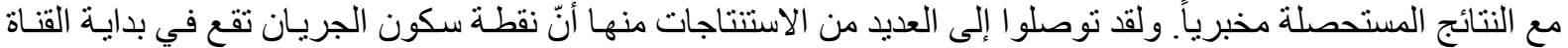

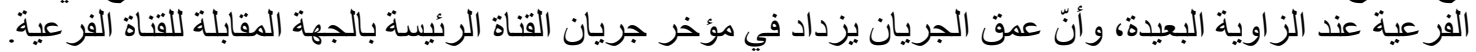

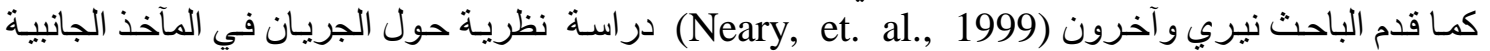

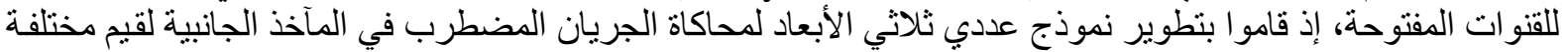

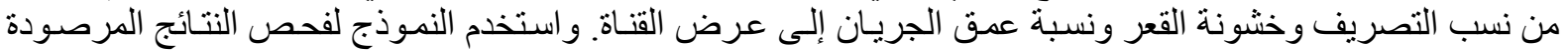

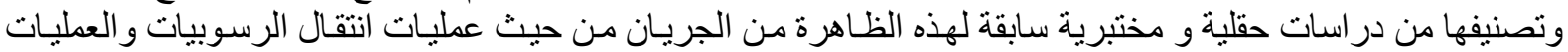

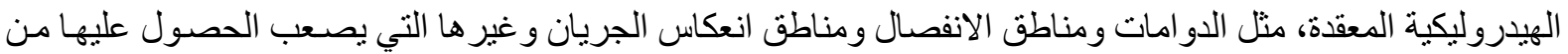

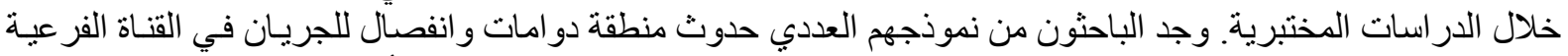

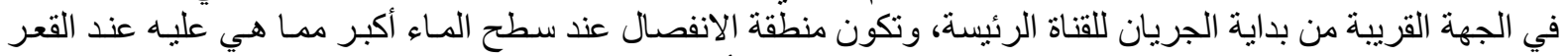

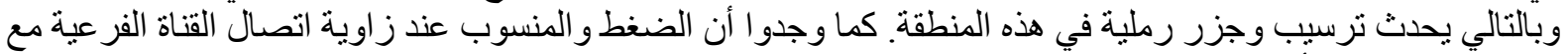

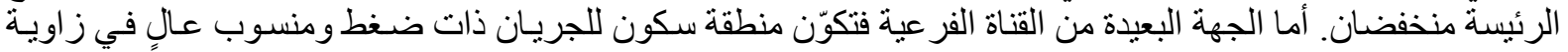

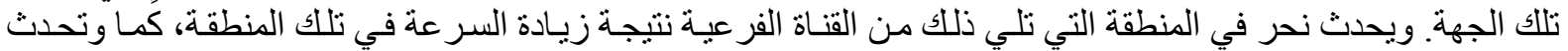

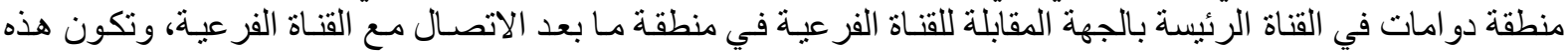
الدوامات غاطسة و اكبر عند قعر القناة. قام كل من الباحث هسو و آخرين (Hsu, et. al., 2002) بدر اسة مختبرية لإيجاد علاقة بين العمق و التصريف،

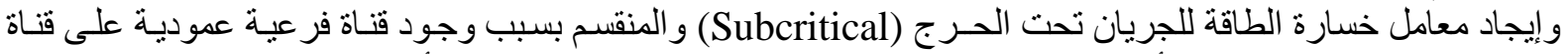

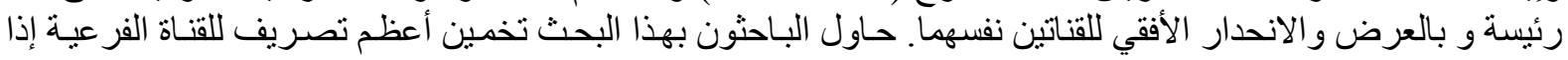

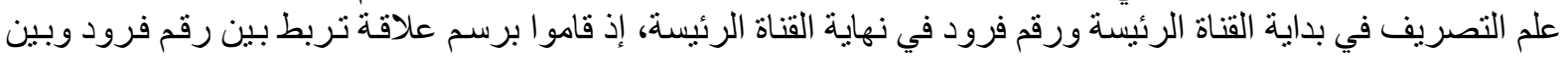

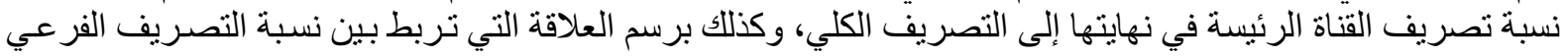

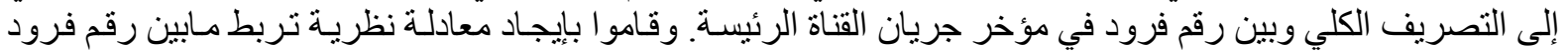

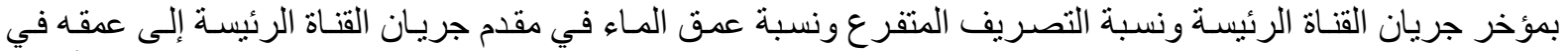

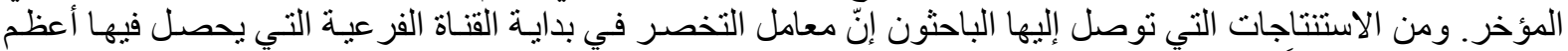

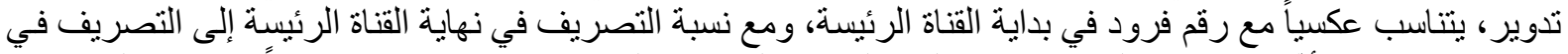

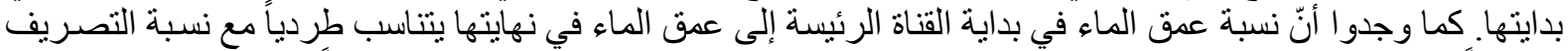

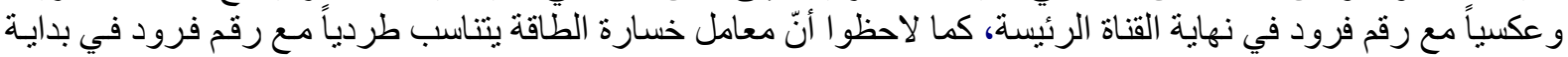

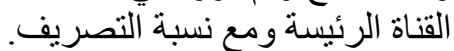

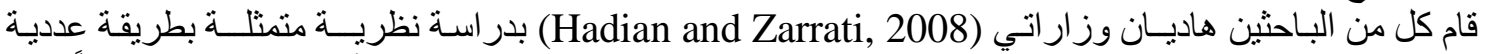

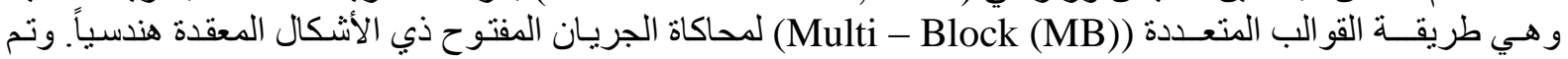

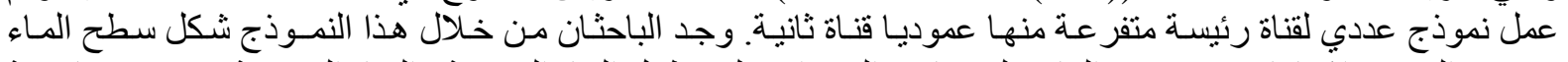

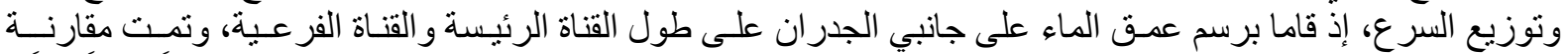

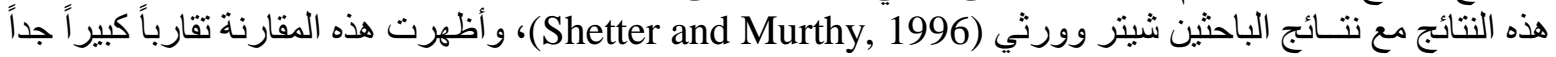


مما أثنتت مدى صلاحية هذا النموذج لإيجاد شكل سطح المـاء في القنوات المفتوحة المعقدة هندسياً مثل القنوات الفر عية وتفر عات الأنهار و وغير ها. النها.

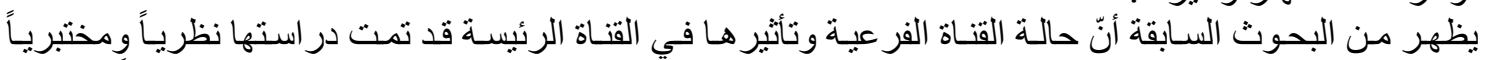

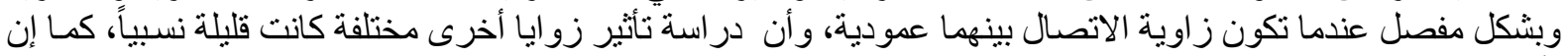

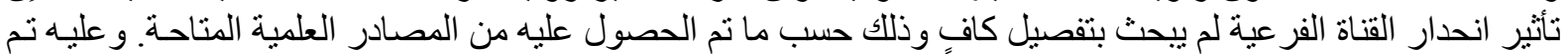
في هذا البحث در اسة تأثير زاوية تفرع القناة الفر عية وانحدار ها في القناة الرئيسة.

\section{التجارب المختبرية}

تم إجر اء التجارب فئي قسم هندسة الموارد المائية في كلية الهندسة / جامعة الموصل باستخدام قناة مختبرية مكونة من جزئين قناة رئيسة وقناة فر عية، وفيما يأتي التفاصيل المتعلقة بهاتين القناتين:

\section{1. القناة الرئيسة والفرعية}

القناة الرئيسة عبارة عن قناة معلقة (Flume)، طولها 10 م و عرضها

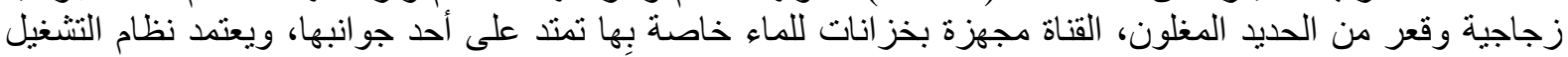

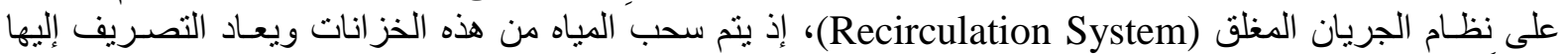

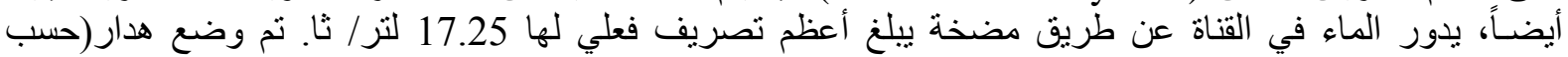

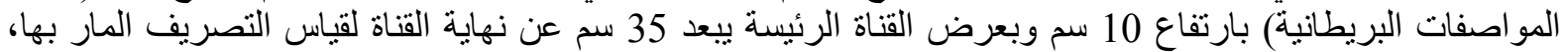

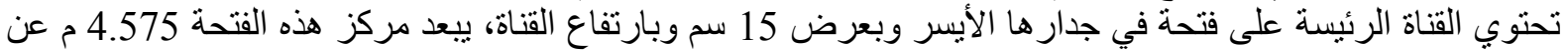

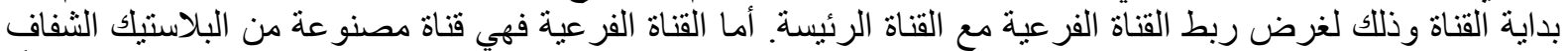

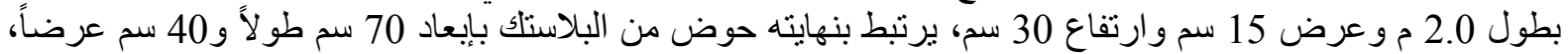

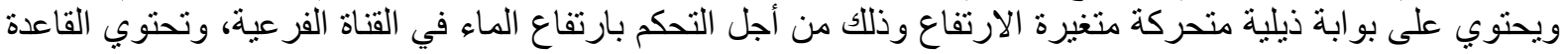

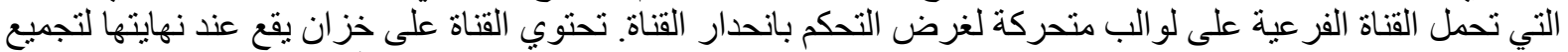

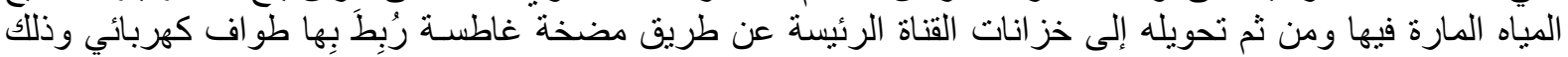

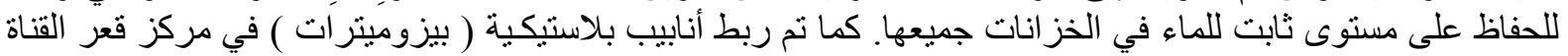

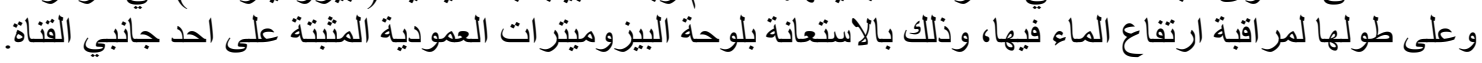

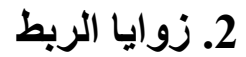

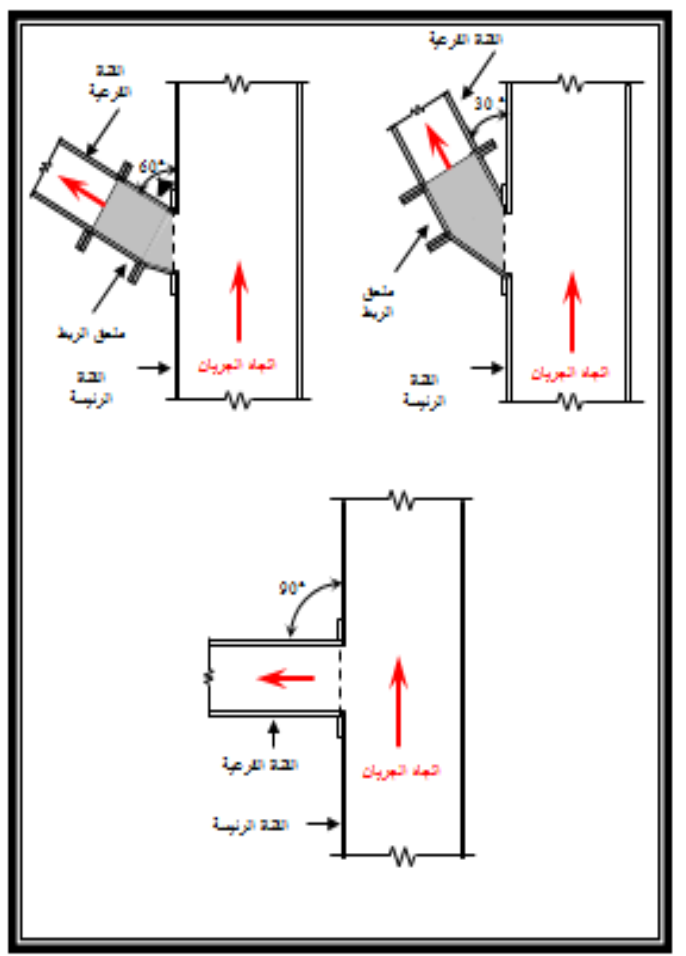

النكل (1): ملحقات الريط و طريقة ريط القناة الفز عبة مع القناة الزئبسة
تم أخذ ثلاث زوايا للقناة الفرعية مع اتجاه الجريان للقناة

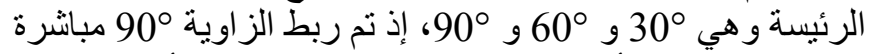

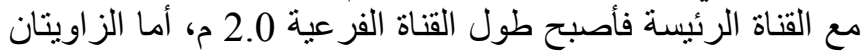
إن $30^{\circ}$ و $60^{\circ}$

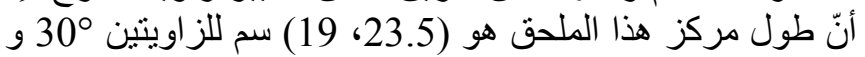
60 $60^{\circ}$ 60² $60^{\circ}$ الربطو طريقة ربط القناة الفرعية مع القناة الرئيسة.

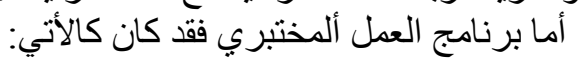

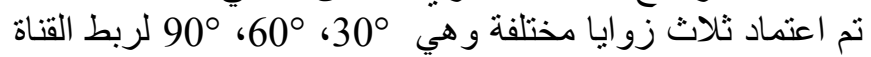

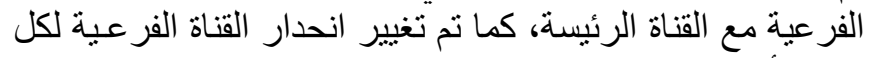

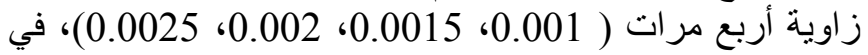

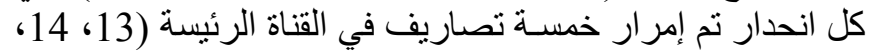

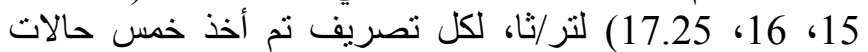
مختلفة لارتفاع الماء في القناة الفرعية وقد نم اختيار ها كما يأتي:

1- أقل ارتفاع ممكن للماء داخل القناة الفر عية دون حدوث تأثير

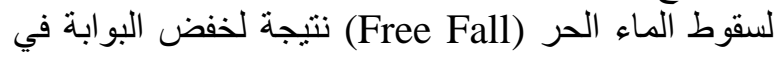

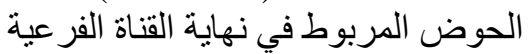

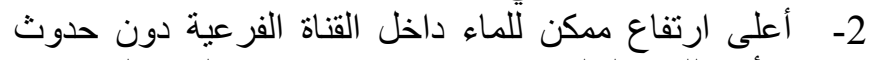

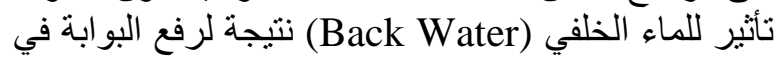
الحوض المربوط بنهاية القناة الفرعية. 3- اختيار قيمة لعمق الماء في القناة بحيث تكون قيمة معامل 


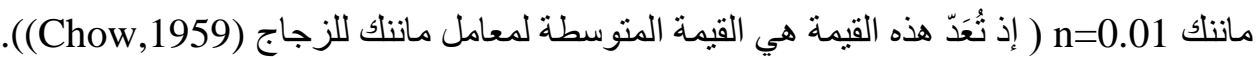
4- تم أخذ عمقين إضافيين للماء بحيث يكون أحدهما أكبر من العمق في النقطة (3) أعلاه، والأخر أقل منه. وبهذا يصبح عدد التجارب المختبرية 300 تجربة. تم قياس منسوب سطح الماء في أثناء التجارب باستخدام المقياس العمودي النقطي (Point Gauge).

تحليل ومناقشة النتائج المختبرية

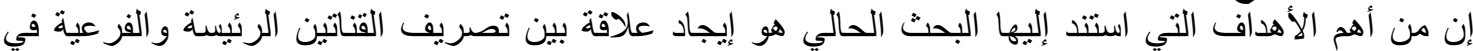
الجريان المتفرع عند تغير انحدار قعر القناة الفرعية وز اوية التقائها مع القناة الرئيسة، لذلك تم إجر اء تحليل شامل للبيانات التئية

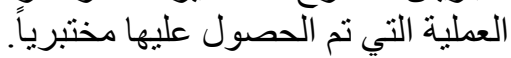

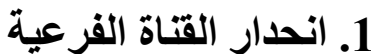

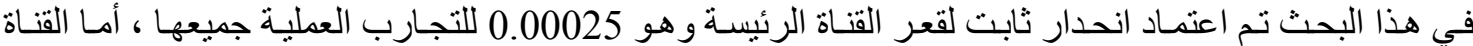
الفرعية فتم أخذ أربع قيم للانحدار وهي

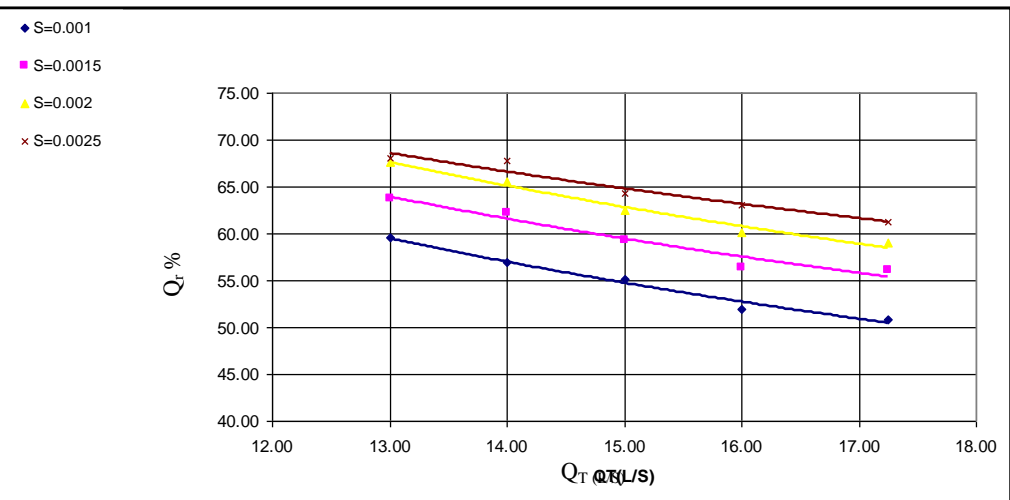

الشكل (2): العلاقة بين نسبة التصريف المتفرع و التصريف الكلي لانحدار اتصري في التصريف فقد تم رسم العلاقة فئة إنين

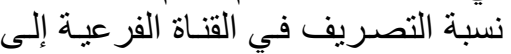

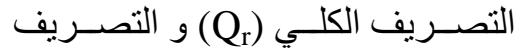

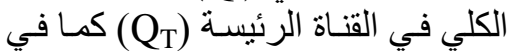
الثــكل (2)، (كنمــوذج للعلاقــة بــين الاثنين)، مع تثبيت ز اوية التفرع وقيمـة

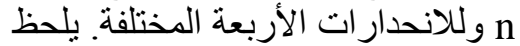
مـن الثـكل السـابق أنّ نسبة التصدية التصريف تزداد مـع زيـادة انحدار القتـاة الفرعيـة الفية

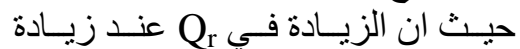

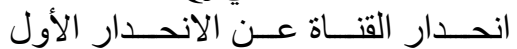
4.25) تراوحـت مـابين (S

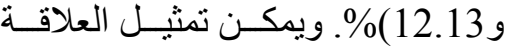
بينهما بالصيغة التالية:

$\mathrm{Q}_{\mathrm{r}} \%=\mathrm{a}_{1} \mathrm{Q}^{-\mathrm{a}_{2}}$

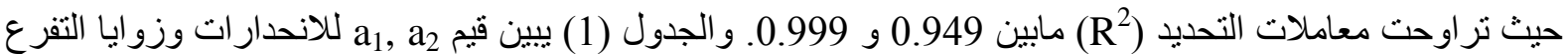
المختلفة و (n=0.01).

الجدول(1): قيم a a a a a المعادلة (1) و لانحدار ات وزوايا تفرع مختلفة (n=0.01)

\begin{tabular}{|c|c|c|c|c|c|c|}
\hline \multirow{2}{*}{$\mathbf{S}_{\mathbf{b}}$} & \multicolumn{6}{|c|}{$\boldsymbol{\theta}$} \\
\cline { 2 - 7 } & \multicolumn{2}{|c|}{$\mathbf{3 0}^{\circ}$} & \multicolumn{2}{|c|}{$\mathbf{6 0}^{\circ}$} & \multicolumn{2}{c|}{$\mathbf{9 0}^{\circ}$} \\
\cline { 2 - 7 } & $\mathbf{a}_{\mathbf{1}}$ & $\mathbf{a}_{\mathbf{2}}$ & $\mathbf{a}_{1}$ & $\mathbf{a}_{2}$ & $\mathbf{a}_{1}$ & $\mathbf{a}_{\mathbf{2}}$ \\
\hline $\mathbf{0 . 0 0 1}$ & 264.33 & 0.5815 & 268.87 & 0.5787 & 252.88 & 0.5598 \\
\hline $\mathbf{0 . 0 0 1 5}$ & 235.97 & 0.5091 & 261.76 & 0.5314 & 252.29 & 0.5207 \\
\hline $\mathbf{0 . 0 0 2}$ & 253.25 & 0.5148 & 241.89 & 0.4831 & 258.19 & 0.5113 \\
\hline $\mathbf{0 . 0 0 2 5}$ & 190.46 & 0.3982 & 246.28 & 0.4779 & 255.52 & 0.4935 \\
\hline
\end{tabular}

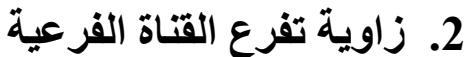

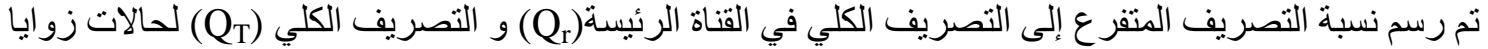

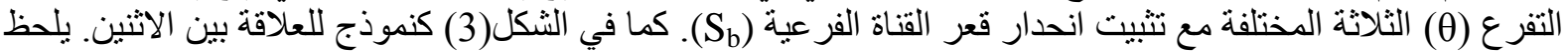




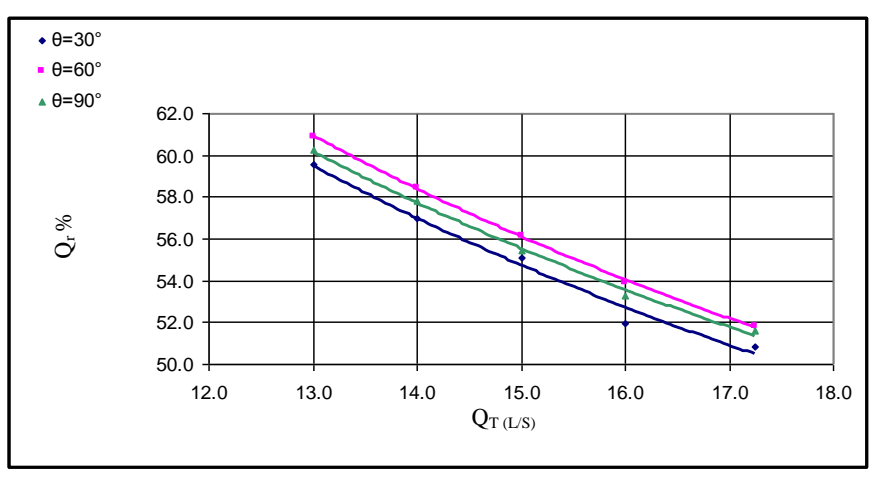

الثكل (3): العلاقة بين نسبة التصريف المتفرع و التصريف

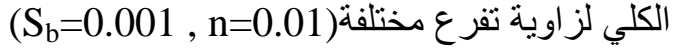

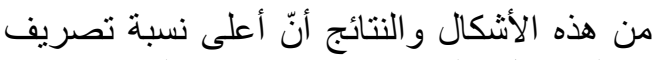

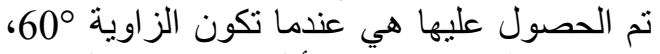

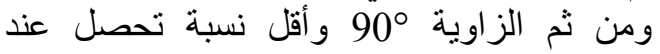

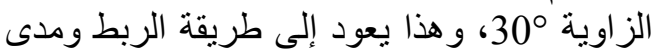

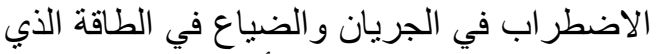
يحدث في منطقة التفرع. إذ أنّ قطعة الربط الطي التي التي

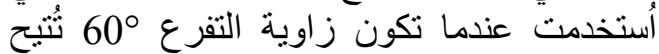
انسيابية أفضل للجريان من القناة الرئيسة إلى القناة

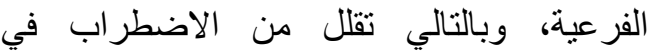

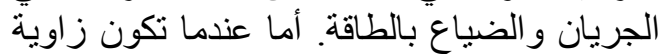

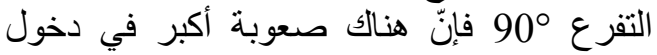

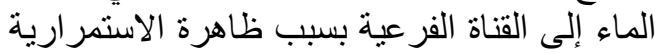

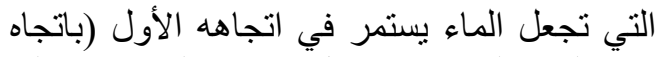

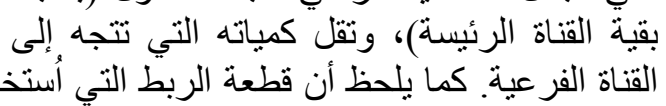

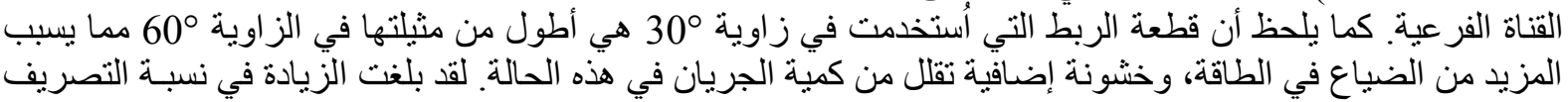

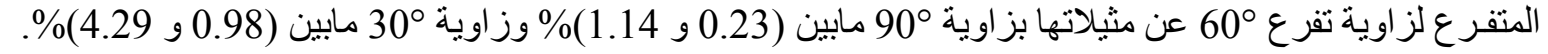

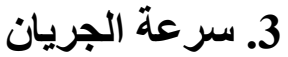

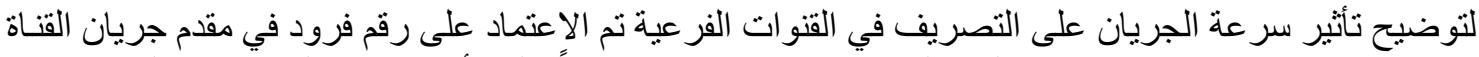

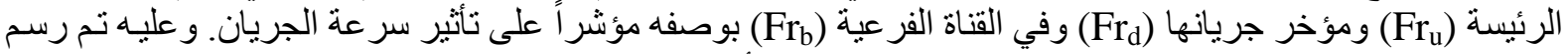

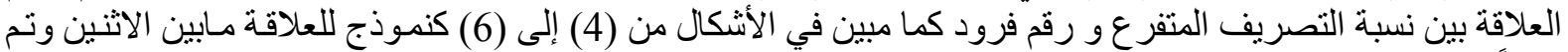

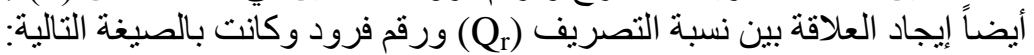

$\mathrm{Q}_{\mathrm{r}} \%=\mathrm{a}_{3}\left(\mathrm{Fr}_{\mathrm{u}}\right)-\mathrm{a}_{4}$

$\mathrm{Q}_{\mathrm{r}} \%=-\mathrm{a}_{5}\left(\mathrm{Fr}_{\mathrm{d}}\right)+\mathrm{a}_{6}$

$\mathrm{Q}_{\mathrm{r}} \%=\mathrm{a}_{7}\left(\mathrm{Fr}_{\mathrm{b}}\right)+\mathrm{a}_{8}$

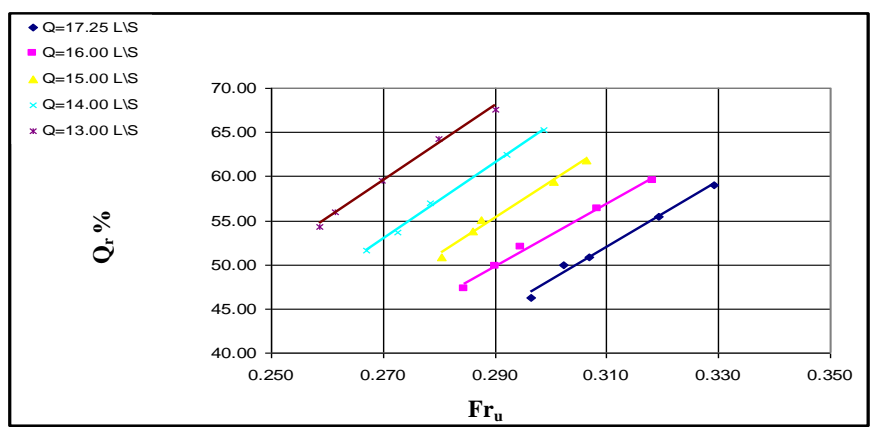

الثكل (4): العلاقة بين نسبة التصريف المتفرع ورقم فرود فى مقدم

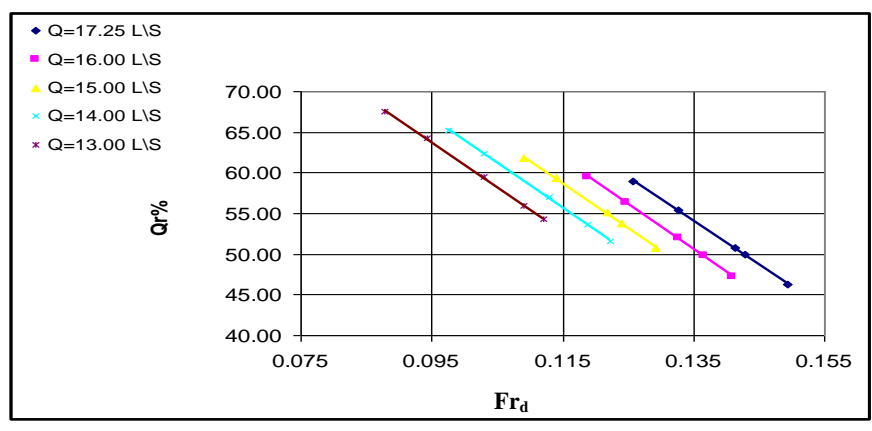

الثكل (5): العلاقة بين نسبة التصريف المتفرع ورقم فرود

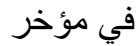

إذ تراوحت معاملات التحديد بين (2) (20.810 و 0.999 ). و الجداول من (2) إلى (4) تبين قيم a a للتصاريف وزو ايا التفرع المختلفة ولحالة الحدار التحار القناة الفرعية بمقدار(0.001) و و (ل)=0.01) بوصنفه نموذج للعلاقة بين الاثنين. ويلحظ من ون الأشكال والمعادلات وجود علاقة خطية قوية بين نسبة

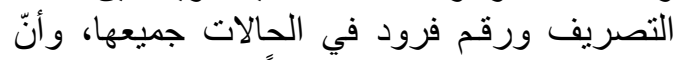

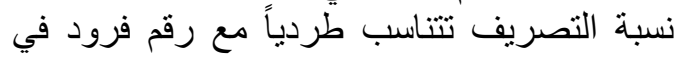
مقدم جريان القناة الرئيسة وفي القناة الفرعية في في في في

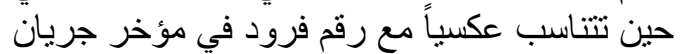

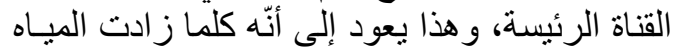

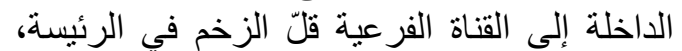

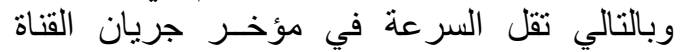

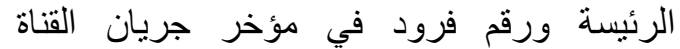
الرئيسة. ومن الجدير بالذكر هنا أنّ الباحث هيد فئ

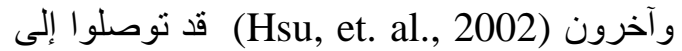
العلاقة العكسية نفسها بين نسبة التصريف ورقات فرود في مؤخر جريان القناة الرئيسة، وأنّ هذه فرده

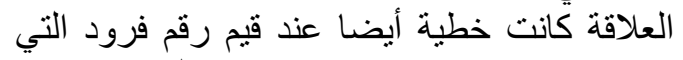
تز اوحت بين (0.0-0.2) كما في هذا البحث. 


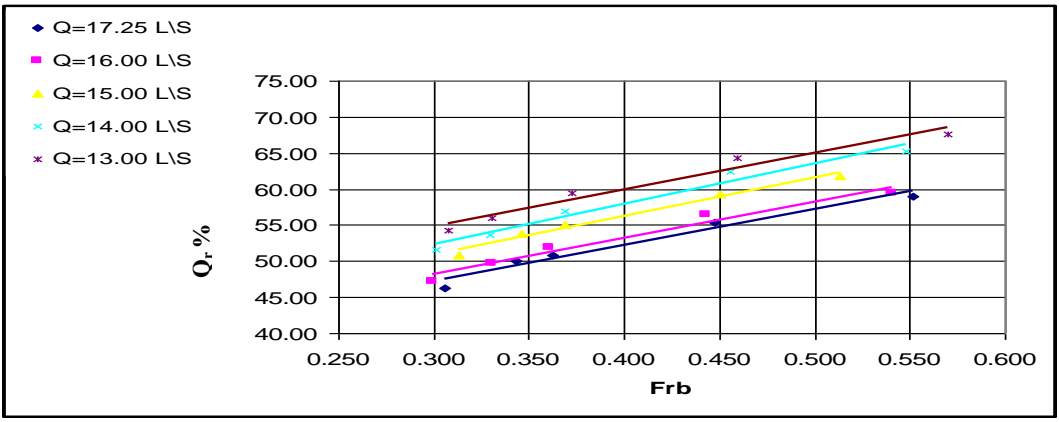

الثكل (6): العلاقة بين نسبة التصريف المتفرع ورقم فرود في

القناة الفرعية (S

الجدول(2): قيم a , a a a المعادلة (2) و للتصاريف وزو ايا التفرع المختلفة (n=0.01, S Sb=0.001)

\begin{tabular}{|c|c|c|c|c|c|c|}
\hline \multirow{2}{*}{\begin{tabular}{l}
\multirow{2}{*}{$\mathbf{L} / \mathbf{S e c}$} \\
\cline { 2 - 7 }
\end{tabular}} & \multicolumn{2}{|c|}{$\mathbf{3 0}_{\mathbf{\circ}}$} & \multicolumn{2}{c|}{$\mathbf{6 0}^{\circ}$} & \multicolumn{2}{c|}{$\mathbf{9 0}^{\circ}$} \\
\cline { 2 - 7 } & $\mathbf{a}_{3}$ & $\mathbf{a}_{\mathbf{4}}$ & $\mathbf{a}_{3}$ & $\mathbf{a}_{\mathbf{4}}$ & $\mathbf{a}_{\mathbf{3}}$ & $\mathbf{a}_{\mathbf{4}}$ \\
\hline $\mathbf{1 3}$ & 424.57 & 55.121 & 385.1 & 44.406 & 403.62 & 50.048 \\
\hline $\mathbf{1 4}$ & 432.69 & 63.959 & 341.98 & 38.646 & 364.96 & 44.919 \\
\hline $\mathbf{1 5}$ & 405.99 & 62.466 & 355.29 & 48.121 & 362.98 & 50.083 \\
\hline $\mathbf{1 6}$ & 356.68 & 53.736 & 401.86 & 66.764 & 359.64 & 53.959 \\
\hline $\mathbf{1 7 . 2 5}$ & 374.79 & 64.184 & 358.31 & 59.499 & 326.06 & 49.311 \\
\hline
\end{tabular}

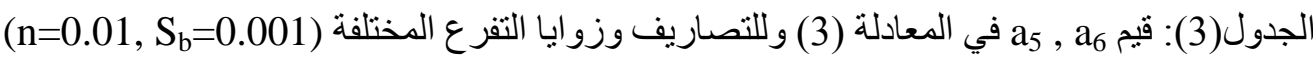

\begin{tabular}{|c|c|c|c|c|c|c|}
\hline \multirow{3}{*}{$\begin{array}{c}\mathbf{Q}_{\mathrm{T}} \\
\mathrm{L} / \mathrm{sec}\end{array}$} & \multicolumn{6}{|c|}{$\boldsymbol{\theta}$} \\
\hline & \multicolumn{2}{|c|}{$30^{\circ}$} & \multicolumn{2}{|c|}{$60^{\circ}$} & \multicolumn{2}{|c|}{$90^{\circ}$} \\
\hline & $\mathbf{a}_{5}$ & $\mathbf{a}_{6}$ & $\mathbf{a}_{5}$ & $a_{6}$ & $\mathbf{a}_{5}$ & $\mathbf{a}_{6}$ \\
\hline 13 & 552.79 & 116.32 & 528.54 & 113.99 & 535.64 & 114.65 \\
\hline 14 & 549.95 & 118.99 & 469.78 & 110.27 & 527.88 & 116.65 \\
\hline 15 & 549.32 & 121.89 & 533.97 & 120.12 & 540.17 & 120.83 \\
\hline 16 & 552.86 & 125.24 & 535.19 & 123.03 & 539.04 & 123.52 \\
\hline 17.25 & 541.24 & 127.2 & 523.38 & 124.8 & 526.34 & 125.2 \\
\hline
\end{tabular}

الجدول(4): قيم a , a a a المعادلة (4) وللتصاريف وزو ايا التفرع المختلفة n=0.01, S Sb=0.001)

\begin{tabular}{|c|c|c|c|c|c|c|}
\hline \multirow{3}{*}{$\begin{array}{c}\mathbf{Q}_{\mathrm{T}} \\
\mathrm{L} / \mathrm{sec}\end{array}$} & \multicolumn{6}{|c|}{$\theta$} \\
\hline & \multicolumn{2}{|c|}{$30^{\circ}$} & \multicolumn{2}{|c|}{$60^{\circ}$} & \multicolumn{2}{|c|}{$90^{\circ}$} \\
\hline & $\mathbf{a}_{7}$ & $\mathbf{a}_{8}$ & $\mathbf{a}_{7}$ & $\mathbf{a s}_{8}$ & $\mathbf{a}_{7}$ & $\mathbf{a}_{8}$ \\
\hline 13 & 51.025 & 39.518 & 65.236 & 36.754 & 58.991 & 37.979 \\
\hline 14 & 56.263 & 35.437 & 60.618 & 36.049 & 54.798 & 37.636 \\
\hline 15 & 53.571 & 34.838 & 62.008 & 32.941 & 53.857 & 35.447 \\
\hline 16 & 50.047 & 33.222 & 60.795 & 31.604 & 59.794 & 31.358 \\
\hline 17.25 & 49.602 & 32.355 & 61.696 & 29.797 & 51.453 & 32.934 \\
\hline
\end{tabular}


خليل: دراسة مختبرية لتأثير زاوية التفرع وانحدار القناة الفرعية على الجريان

الجدول(5): قيم a9, a في المعادلة (5) وللتصساريف وزو ايا التفرع المختلفة (n=0.01, S S =0.001)

\begin{tabular}{|c|c|c|c|c|c|c|}
\hline \multirow{3}{*}{$\begin{array}{c}\mathbf{Q}_{\mathrm{T}} \\
\mathbf{L} / \mathbf{s e c}\end{array}$} & \multicolumn{6}{|c|}{$\theta$} \\
\hline & \multicolumn{2}{|c|}{$30^{\circ}$} & \multicolumn{2}{|c|}{$60^{\circ}$} & \multicolumn{2}{|c|}{$90^{\circ}$} \\
\hline & $a_{9}$ & $\mathbf{a}_{10}$ & $\mathbf{a}_{9}$ & $\mathbf{a}_{10}$ & $\mathbf{a}_{9}$ & $\mathbf{a}_{10}$ \\
\hline 13 & 82.869 & 130.48 & 135.6 & 182.68 & 111.42 & 158.33 \\
\hline 14 & 98.019 & 141.4 & 105.39 & 152.6 & 101.44 & 147.71 \\
\hline 15 & 87.604 & 130.68 & 119.75 & 161.62 & 87.18 & 131.97 \\
\hline 16 & 81.987 & 121.57 & 113.97 & 154.54 & 113.22 & 152.17 \\
\hline 17.25 & 84.105 & 123.17 & 129.97 & 167.91 & 93.576 & 133.97 \\
\hline
\end{tabular}

4. تسبة عمق نق الماء

تم رسم قيم نسبة التصريف المتفرع إلى التصريف الرئيس و نسبة عمق الماء في القناة الفرعية إلى عمقه في مؤخر

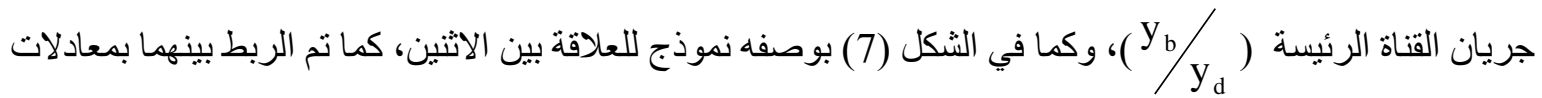
خطيـة بسيطة بالصيغة التالية:

$\mathrm{Q}_{\mathrm{r}} \%=-\mathrm{a}_{9}\left(\mathrm{y}_{\mathrm{b}} / \mathrm{y}_{\mathrm{d}}\right)+\mathrm{a}_{10}$

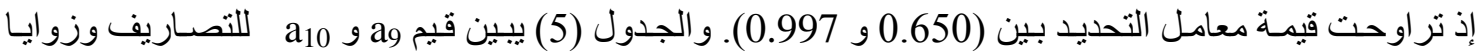

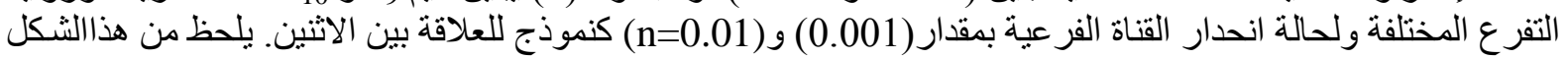

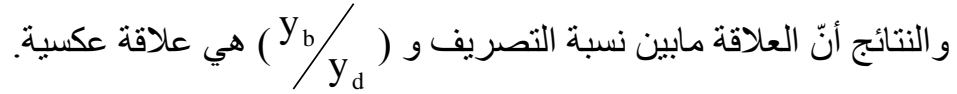

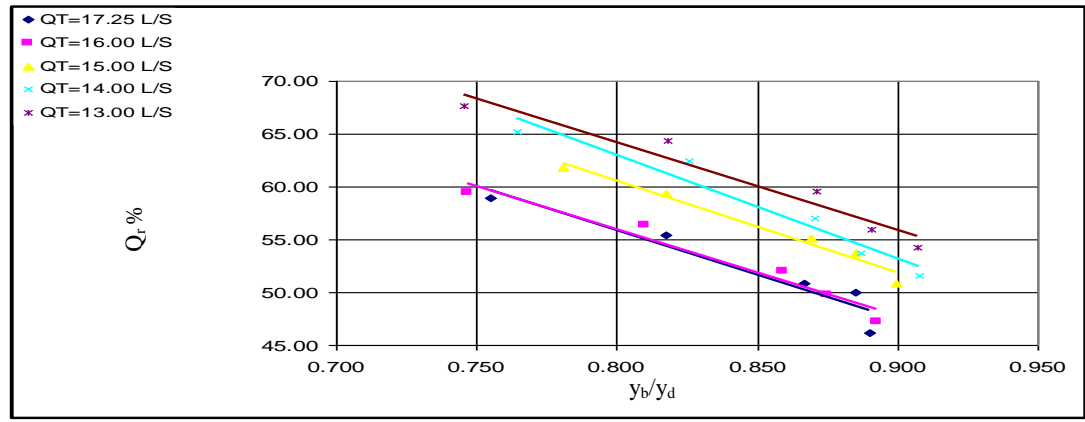

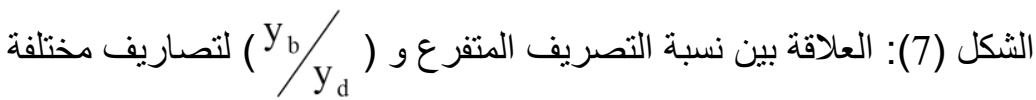

$$
\left(\theta=30^{\circ}, S_{h}=0.001\right)
$$

إيجاد معادلة وضعية لحساب نسبة التصريف المتفرع الإدئ

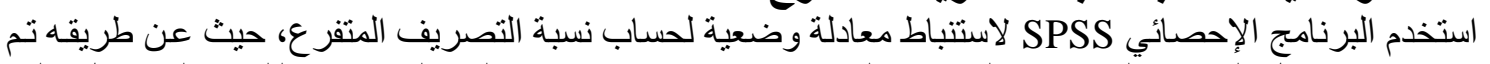

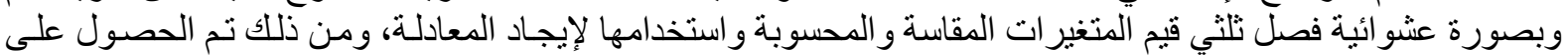
$\mathrm{Qr} \%=27.98 \cdot\left(\frac{\mathrm{S}_{\mathrm{b}}{ }^{0.029} \cdot(\sin \theta)^{0.053}}{\left(\frac{\mathrm{y}_{\mathrm{b}}}{\mathrm{y}_{\mathrm{d}}}\right)^{0.384} \cdot \mathrm{Fr}_{\mathrm{d}}{ }^{0.409}}\right)$ العلاقة الآتية وبمعامل تحديد (R2) مقداره (0.953) 
لقد تم استخدام الثلث المتبقي من قيم المتغير ات للتحقق من دقة المعادلة (6)، إذ تم أيضـاً حسـاب نسبة الخطأ لهذه القيم من

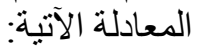

$\%$ Error $=\left(\frac{\left(\mathrm{Q}_{\mathrm{r}}\right)_{\text {act. }} \%-\left(\mathrm{Q}_{\mathrm{r}}\right)_{\text {the. }} \%}{\left(\mathrm{Q}_{\mathrm{r}}\right)_{\text {act. }} \%}\right) \times 100$

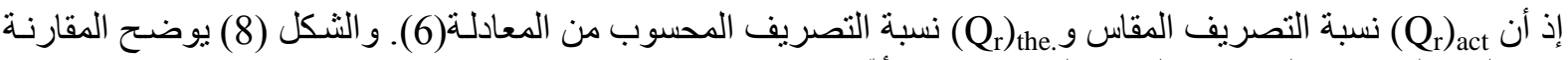

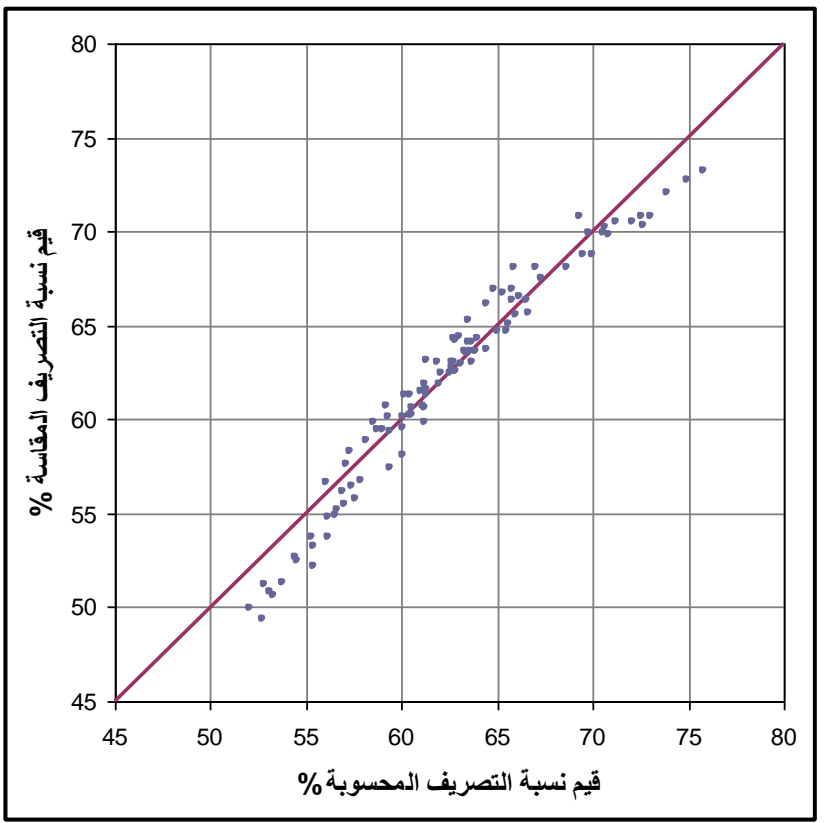

الثكل (8): المقارنة بين القيم المقاسة و المحسوبة لنسبة

$$
\text { التصريف }
$$

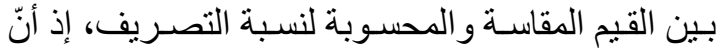

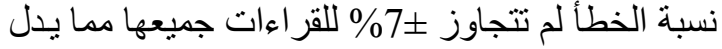

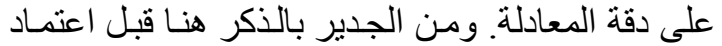

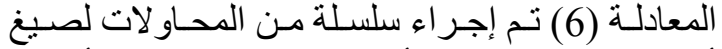

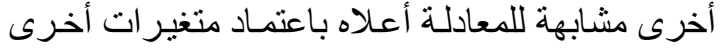
يعتقد بوجود تأثير مباشر ومهم للها لهاعلى على نسبة التصريف

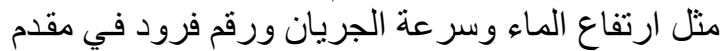

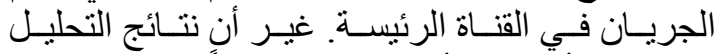

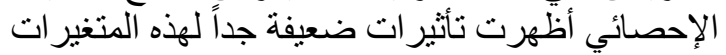

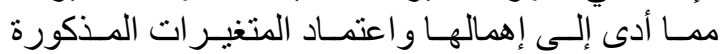
بالمعادلة (6) بدلاً عنها.

الاستنتاجات

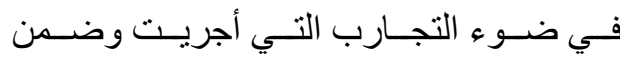
محددات هذا البحث نم التوصل إلى الاستنتاجات التالية:

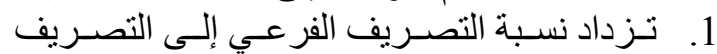

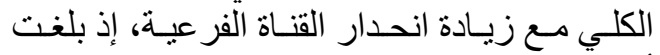

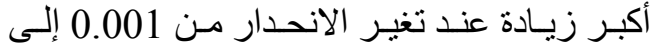
. 0.0025 2. أعلى نسبة تصريف تم الحصول عليها هي عندما

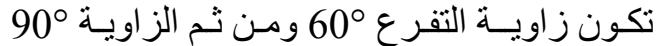

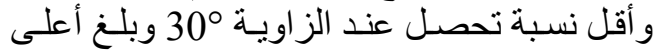

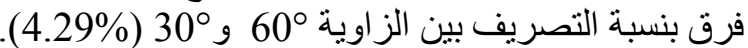

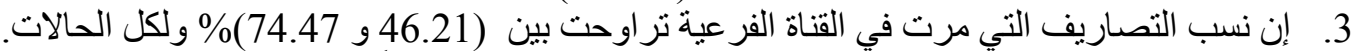

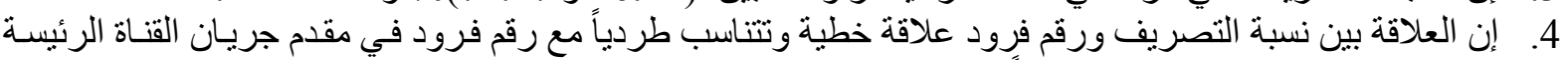

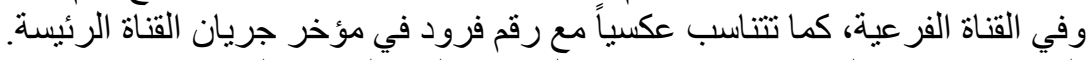

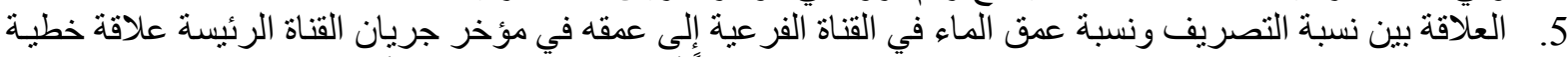

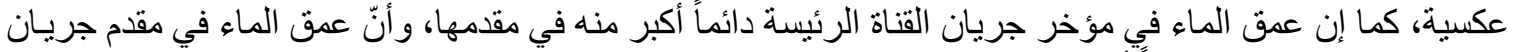
القناة الرئيسة ومؤخر ها دائماً أكبر من عمق في المئ الماء في القناة الفر عية.

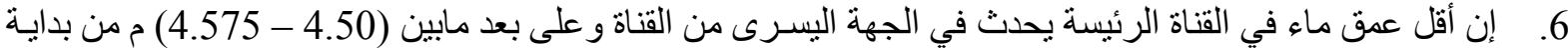

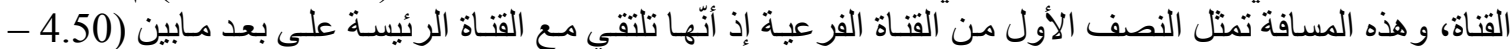

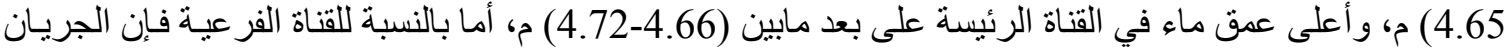

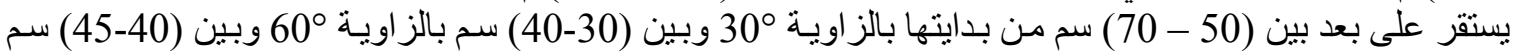

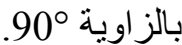
7. إن التغير الحاصل بنسبة التصـريف قلبل نسبياً عند تغير زاويـة التفر ع في حـال بقاء مدخل القناة الفرعية مسـاوياً

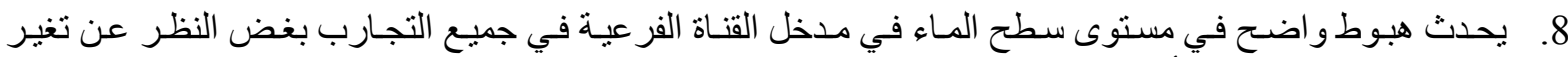

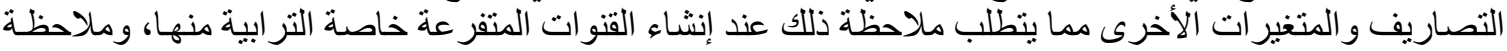

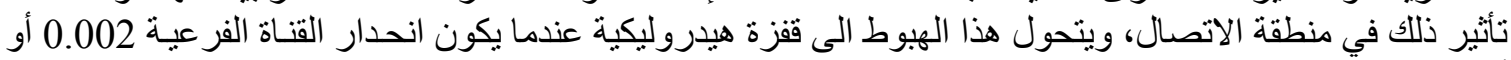




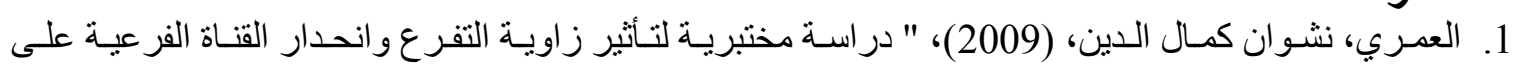
الجريان" رسالة ماجستير، هندسة الموارد المائية / هيدروليك، كلية الهندسة ، جامعة الموصل.

2. British Standard Institution (BSI), (1965), "Method of Measurement of Liquid Flow in Open Channel', part 4A.BSI, 3680,London,English,U.K.

3. Chow, V. T., (1959), "Open Channel Hydraulics”, Mc Crow-Hill book Co., New York.

4. Hadian, M. R., and Zarrati, A. R., (2008), “Application of Multi-Block Method for Simulating Shallow Free Surface Flows In Complex Geometries", Journal of Hydraulic Research, Vol. 46 No. 5, pp. 668-678.

5. Hsu, C.C., Tang, C.J., Lee, W. J. and Shieh, M. Y., (2002), "Subcritical $90^{\circ}$ Equal Width. Open - Channel Dividing Flow', ASCE, J. of Hydraulic Engineering, Vol. 128, No.7, pp. 716-720.

6. Neary, V.S., Sotiropoulos, F. and Odgaard, A.J., (1999), "Three-Dimensional Numerical Model of Lateral-Intake Inflows", ASCE, J. of Hydraulic Engineering, Vol. 125, No.2, pp. 126-140.

7. Ramamurthy, A.S., Tran, D.M. and Carballada, L. B., (1990), “Dividing Flow in Open Channels", ASCE, J. of Hydraulic Engineering, Vol. 116, No.7, pp. 449-455.

8. Shettar, A. S., and Murthy, K. K. (1996). "A Numerical Study of Division of Flow in Open Channels', J. Hydraulic Res., Vol. 34, No. 5, pp. 651-675.

$$
\text { تم اجراء البحث في كلية ألهنسة = جامعة ألموصل }
$$

